\title{
The generation of non aspect sensitive plasma density irregularities by field aligned drifts in the lower ionosphere
}

\author{
T. R. Robinson ${ }^{1}$, K. Schlegel ${ }^{2}$ \\ ${ }^{1}$ University of Leicester, Leicester, UK \\ e-mail: txr@ion.le.ac.uk \\ ${ }^{2}$ Max-Planck-Institut für Aeronomie, Katlenburg-Lindau, Germany
}

Received: 19 August 1999 / Revised: 3 December 1999 / Accepted: 6 December 1999

\begin{abstract}
A theory of the generation of plasma density irregularities with virtually no aspect sensitivity, in the lower ionosphere at high latitudes, by electron drifts aligned with the geomagnetic field, is presented. The theory is developed through fluid equations in which the destabilising mechanism involves positive feedback from electron collisional heating. When field aligned electron drift speeds exceed a few $\mathrm{km} \mathrm{s}^{-1}$, this effect destabilises waves with wavelengths in excess of a few tens of metres in the lower E-region, where collisional effects are sufficiently large. Furthermore, the threshold conditions are almost independent of the wave propagation direction and the unstable waves propagate at speeds well below the ion acoustic speed. The role that this new instability may play in recent radar backscatter observations of short scale irregularities propagating in directions close to that of the geomagnetic field, in the lower E-region is also considered.
\end{abstract}

Key words: Ionosphere (auroral ionosphere; ionospheric irregularities; plasma waves and instabilities)

\section{Introduction}

Small-scale plasma density irregularities are an important and ubiquitous phenomenon in the ionospheric E-region at altitudes below $130 \mathrm{~km}$. Those with sufficiently short wavelengths commonly give rise to strong coherent radar backscatter in the HF, VHF and even the UHF bands, when the radar beams are directed orthogonal to the geomagnetic field. The irregularities responsible for this strong, nearly orthogonal, scatter are elongated along the geomagnetic field direction with

Correspondence to: T. R. Robinson their $\mathbf{k}$ vectors orientated within a fraction of a degree of a direction orthogonal to the field and parallel to the radar wave vector. A number of different plasma instabilities are capable of generating small-scale field aligned plasma density irregularities in the lower ionosphere at high latitudes, but probably the most important of these, especially for irregularity wavelengths between one and a few tens of metres is the wellknown Farley-Buneman instability (FBI, Farley, 1963; Buneman, 1963). The main characteristics which distinguish the FBI are that it requires the difference in the electron and ion velocities across the geomagnetic field, caused by $\mathbf{E} \times \mathbf{B}$ drifts, to exceed the local ion-acoustic speed. Also, the phase speed of the marginally unstable waves is equal to the ion-acoustic speed in the fluid limit.

Although the majority of coherent radar observations indicate backscatter within a degree or so of orthogonality, in agreement with FBI theory, Rüster and Schlegel (1999) have now reported strong backscatter observations in an altitude range of $90-100 \mathrm{~km}$, with a $50 \mathrm{MHz}$ radar beam pointing almost parallel to the geomagnetic field direction, i.e. at aspect angles close to $90^{\circ}$. Thus, the density irregularities responsible for this backscatter would have to have their $\mathbf{k}$ vectors almost parallel to the geomagnetic field direction. The observations of Rüster and Schlegel (1999) also appear to indicate scatter from waves travelling at speeds well below the local ion sound speed. These new observations by Rüster and Schlegel (1999) have motivated the present search for an instability which can excite irregularities propagating in the geomagnetic field direction, in the lower E-region, where collisions between plasma particles and neutrals are important.

Now, it is well known that in collisionless plasmas, such as exist in the topside ionosphere, electron drifts parallel to the geomagnetic field with values in excess of a few times the ion acoustic speed can give rise, through kinetic effects, to the ion acoustic instability (Kindel and Kennel, 1971). This instability involves the excitation of ion acoustic waves propagating in a direction essentially parallel to the geomagnetic field. Indeed, evidence from 
radar backscatter experiments that such waves are excited at altitudes above $140 \mathrm{~km}$ in the auroral ionosphere has recently been reported (Rietveld et al., 1991). In this altitude range, both ions and electrons are effectively collisionless. However, this instability cannot operate in the highly collisional conditions at altitudes well below $130 \mathrm{~km}$, where ion dynamics in particular become dominated by collisions with neutrals, except at very short wavelengths of a few centimetres. Only radars operating at frequencies above several $\mathrm{GHz}$ would be sensitive to such waves (see the Appendix for details of the limits of the collisionless approximation). A further point against this instability as an explanation for Rüster and Schlegel's (1999) observations is that it requires the electron temperature to greatly exceed that of the ions and furthermore the phase speed of the waves corresponds to the ion acoustic speed in this heated plasma. Neither of these two conditions is consistent with Rüster and Schlegel's (1999) report. However, there is evidence that field aligned currents were present during the large aspect angle backscatter events reported by Rüster and Schlegel (1999) and thus may play a role in exciting the irregularities responsible for their observations.

Field aligned currents play an important role in ionosphere-magnetosphere coupling at high latitudes and continue to be a major topic of research. Much important information about them, particularly with regard to where and how they close in the lower ionosphere remains illusive. There are no direct observations in the lower ionosphere of the strength of the field aligned currents and, more crucially in the present context, of the size of the directed field aligned velocities associated with them. However, strong indirect evidence that these currents penetrate into the lower ionosphere has been reported in the literature. In particular, field aligned currents have been invoked, in order to explain the excitation of small aspect angle irregularities at thresholds below those expected for the FBI with $\mathbf{E} \times \mathbf{B}$ drifts alone (Chaturvedi et al., 1987). Villain et al. (1987) also used similar arguments in the case of HF radar backscatter observations from electrostatic ioncyclotron waves. Chaturvedi et al. (1987), who based their parallel velocity considerations on spacecraft observations of field aligned currents by Bythrow et al. (1984), estimated that electron drifts of up to $40 \mathrm{~km} \mathrm{~s}^{-1}$ parallel to the geomagnetic field could occur at altitudes of around $105 \mathrm{~km}$. It should be pointed out, here, that these altitudes are somewhat arbitrary, since the height the backscatter is not well determined in radar experiments. There is probably a margin of uncertainty in the altitude of backscattering irregularities of around a neutral scale height (i.e. approximately 5 to $10 \mathrm{~km}$ ). Haldoupis et al. (1994) have also pointed out the importance of field aligned currents in the explanation of large aspect angle radar backscatter observations, at angles much larger than predicted from the linear instability theory of the FBI (although these were still much smaller than those reported by Rüster and Schlegel, 1999). Haldoupis et al. (1994) utilised a theoretical model of Hamza (1992), who invoked field aligned currents in the lower E-region as the origin of anomalous collisions due to a very short scale ionacoustic instability.

Although, in principle, the FBI could excite irregularities at large aspect angles, such as those reported by Rüster and Schlegel (1999), under the influence of field aligned electron drifts alone, the threshold drifts required exceed those proposed by Chaturverdi et al. (1987) by more than an order of magnitude. Furthermore, these large parallel electron drifts would inevitably give rise to enormous levels of electron heating which would raise the electron temperature to values many tens of times that of the ions. Such effects have never been observed at altitudes below $100 \mathrm{~km}$ and are highly unlikely to occur there. Moreover, the resulting enhancements in the electron temperature would so strongly stabilise the plasma wave, by raising the local ion acoustic speed, that the instability could not in fact be excited under these conditions (see Sect. 2). Thus, the FBI can also probably be ruled out as a convincing explanation for the observations of Rüster and Schlegel (1999).

Dimant and Sudan (1995) have recently developed a kinetic theory which predicts that low-frequency field aligned waves are destabilised in the lower E-region due to electron thermal effects, in the presence of $\mathbf{E} \times \mathbf{B}$ drifts. It has subsequently been demonstrated that this instability can also be adequately treated using fluid theory, as long as the electron energy balance and heat flow effects are included (Dimant and Sudan, 1997; Robinson, 1998). The destabilising mechanism for the Dimant and Sudan instability (DSI) is electron-neutral frictional heating due to electron motion through essentially stationary background neutrals, under the influence of convection electric fields which drive $\mathbf{E} \times \mathbf{B}$ drifts. The DSI, like the FBI, still results in strongly aspect sensitive, almost field aligned irregularities and is not suitable as an explanation of the observations of Rüster and Schlegel (1999).

A new theory of plasma irregularity excitation at large aspect angles by electron-neutral frictional heating effects due to field aligned electron flows is developed in this work. The main difference between the present theory and that of Dimant and Sudan $(1995,1997)$ is that whereas the electron Pedersen conductivity is responsible for the frictional heating in the latter case, it is the parallel conductivity in the former case. This new theory predicts that, in contrast to what is found in the case of either the FBI or the DSI, the phase speed of the marginally unstable plasma irregularities, travelling in a direction parallel to the geomagnetic field, is more than an order of magnitude below the ion-acoustic speed. Furthermore, the size of the field aligned electron flow required to destabilise these instabilities is only a few $\mathrm{km} \mathrm{s}^{-1}$, which is not only well below previously proposed values (e.g. Chaturvedi et al., 1987; Villain et al., 1987), but which is also low enough not to cause very large electron temperature enhancements, although moderate heating does occur. In addition, the threshold values of the flows are largely independent of aspect angle. Whilst these characteristics are also consistent 
with a number of aspects of the observations of Rüster and Schlegel (1999), the instability presented here is confined to wavelengths longer than a few tens of metres and probably cannot explain directly the backscatter at the shorter radar wavelengths utilised by these authors. However, as discussed in the concluding section, it may be possible, with the inclusion of certain additional nonlinear effects, to devise an explanation of Rüster and Schlegel's observations based on the present theory. A complex dispersion relation for this new instability is developed in the following section.

\section{Mathematical development}

The starting point for the analysis are the fluid equations of electron continuity, momentum and energy conservation and heat flow, as follows (Golant et al., 1980)

$$
\begin{aligned}
& \partial N_{e} / \partial t+\nabla \cdot\left(N_{e} \mathbf{V}_{e}\right)=0 \\
& N_{e} m_{e}\left(\partial \mathbf{V}_{e} / \partial t+\mathbf{V}_{e} \cdot \nabla \mathbf{V}_{e}\right) \\
& =-N_{e} e\left(\mathbf{E}+\mathbf{V}_{e} \wedge \mathbf{B}\right)-\nabla N_{e} T_{e}-N_{e} m_{e} v_{e n} \mathbf{V}_{e} \\
& 1.5\left(\partial N_{e} T_{e} / \partial t+\mathbf{V}_{e} \cdot \nabla N_{e} T_{e}\right)+2.5 N_{e} T_{e} \nabla \cdot \mathbf{V}_{e}+\nabla \cdot \mathbf{q}_{e} \\
& =N_{e} m_{e} \delta_{e} v_{e n}\left(T_{n}-T_{e}\right)+N_{e} m_{e} v_{e n} \mathbf{V}_{e}^{2} \\
& \partial \mathbf{q}_{e} / \partial t+\mathbf{V}_{e} \cdot \nabla \mathbf{q}_{e}=-\Omega_{e} \mathbf{q}_{e} \wedge \mathbf{b}-v_{e n} \mathbf{q}_{e}
\end{aligned}
$$

where $m_{e}, N_{e}, T_{e}, \mathbf{V}_{e}$ and $\mathbf{q}_{e}$ are the electron mass, number density, temperature, velocity and heat flux, respectively. Also, $T_{n}$ is the neutral temperature and $v_{e n}$ and $\delta_{e} v_{e n}$ represent the elastic momentum exchange and inelastic energy exchange electron-neutral collision frequencies, respectively. $\mathbf{E}$ and $\mathbf{B}$ are respectively the electric field and the magnetic flux density and $\mathbf{b}$ is a unit vector along $\mathbf{B}$. The second term on the right hand side of Eq. (3) is the electron collisional heating term. Also above, $\Omega_{e}\left(=e B / m_{e}\right)$ is the electron gyro-frequency. In addition to the electron dynamics, above, ion dynamics, sufficient for present purposes, are included with the aid of the ion continuity and momentum conservation equations that follow. The variables involved correspond to those in Eqs. (1) to (4), but with subscript ' $i$ ' in place of ' $e$ ' for the ions rather than the electrons.

$\partial N_{i} / \partial t+\nabla \cdot\left(N_{i} \mathbf{V}_{i}\right)=0$

$N_{i} m_{i}\left(\partial \mathbf{V}_{i} / \partial t+\mathbf{V}_{i} \cdot \nabla \mathbf{V}_{i}\right)=N_{i} e \mathbf{E}-\nabla N_{i} T_{i}-N_{i} m_{i} v_{i n} \mathbf{V}_{i}$

In Eqs. (2) and (6) account is taken of the fact that the electrons are magnetised whilst the ions are not, in the E-region, and hence, $v_{e n} \ll \Omega_{e}$ and $v_{i n} \gg \Omega_{i}\left(=e B / m_{i}\right)$, where $v_{i n}$ and $\Omega_{i}$ are respectively the ion-neutral collision frequency and the ion-gyro frequency. Furthermore, velocities are all referred to a frame of reference stationary with respect to the neutrals. Also, given that the ions are unmagnetised, their steady state velocity can be considered to be the same as that of the neutrals.

A sinusoidal wave perturbation analysis of Eqs. (1) to (6) yields the following set of first order Equations.

$$
\begin{aligned}
& n_{1} \omega_{e}-\mathbf{k} \cdot \mathbf{V}_{e 1}=0 \\
& \left(v_{e n}-i \omega_{e}\right) \mathbf{V}_{e 1} / \Omega_{e}+\mathbf{V}_{e 1} \wedge \mathbf{b}-i \mathbf{k} \phi_{1} / B \\
& +i \mathbf{k}\left(T_{e 0} / m_{e}\right)\left(n_{1}+t_{1}\right)+\alpha v_{e n} \mathbf{V}_{e 0} t_{1} / \Omega_{e}=0 \\
& i \omega_{e} n_{1}+\left(-1.5 i \omega_{e}+\delta_{e}^{*} v_{e n}\right) t_{1}+i \mathbf{k} \cdot \mathbf{q}_{e 1} /\left(N_{e 0} T_{e 0}\right) \\
& -2 m_{e} v_{e n} \mathbf{V}_{e 1} \cdot \mathbf{V}_{e 0} / T_{e 0}=0 \\
& \left(v_{e n}-i \omega_{e}\right) \mathbf{q}_{e 1} / \Omega_{e}+\mathbf{q}_{e 1} \wedge \mathbf{b}+2.5 i N_{e 0} T_{e 0}^{2} \mathbf{k} t_{1} /\left(m_{e} \Omega_{e}\right)=0
\end{aligned}
$$

$n_{1} \omega-\mathbf{k} \cdot \mathbf{V}_{i 1}=0$

$\left(v_{i n}-i \omega\right) \mathbf{V}_{i 1} / \Omega_{i}+i \mathbf{k} \phi_{1} / B+i \mathbf{k}\left(T_{i 0} / m_{i} \Omega_{i}\right) n_{1}=0$

where $\omega$ and $\mathbf{k}$ are the wave frequency (in the frame of the neutrals) and the wave vector respectively. Also, $\omega_{e}=\omega-\mathbf{k} \cdot \mathbf{V}_{e 0}$ is the electron wave frequency in the frame of the neutrals. Since the electric field fluctuations are entirely electrostatic, $\mathbf{E}_{1}=-i \mathbf{k} \phi_{1}$, where, $\phi_{1}$ is the electric potential perturbation. Subscript 0 indicates steady background values, whilst subscript 1 indicates first order field variables. It is also assumed that the plasma is quasi-neutral and hence the electron and ion densities are assumed equal. Further, $n_{1}=N_{1} / N_{e 0}$ and $t_{1}=T_{e 1} / T_{e 0}$. It should be noted that the ion fluid equations, Eqs. (10) and (11), are only valid if $v_{i n} \gg k \sqrt{T_{i 0} / m_{i}}$ (see the Appendix for further details). Furthermore, under these conditions ion thermal effects are negligible and hence there is no need for an ion energy balance equation. For the electrons a similar constraint applies, i.e. $v_{e n} \gg k \sqrt{T_{e 0} / m_{e}}$. However, wavelength constraints for the electron case are much more complicated than that for the ions, since thermal electron effects are crucial to the behaviour of the waves under consideration and these will be dealt with in more detail later.

In Eq. (8), the coefficient, $\alpha$ takes account of the temperature dependence of the electron elastic momentum exchange collision frequency through the relation $v_{e n}\left(T_{e}\right)=v_{e n}\left(T_{e 0}\right)\left(T_{e} / T_{e 0}\right)^{\alpha}$. Also, in Eq. (9), $\delta_{e}^{*}$ is given by

$\delta_{e}^{*}=\delta_{e}\left(T_{e 0}\right)+\left(\partial \delta_{e} / \partial T_{e}\right)\left(T_{e 0}-T_{i 0}\right)$

In what follows it is assumed that

$|\cos \theta| \gg\left|\left(v_{e n}-i \omega_{e}\right) / \Omega_{e}\right|$

where $\theta$ is the angle between the wave vector and the geomagnetic field. The physical implication of Eq. (14) is that, with propagation angles in these directions, the electron fluctuations velocity is along the magnetic field direction.

Eliminating first order field variables from Eqs. (7) to (12), under the condition specified by Eq. (14), then yields the dispersion relation

$$
\begin{aligned}
& \omega_{e}\left(v_{e n}-i \omega_{e}\right) / \Omega_{e}+\left\{(\mathbf{k} \cdot \mathbf{b} / k)^{2}\left(\omega\left(v_{i n}-i \omega\right)+i k^{2} c^{2}\right) / \Omega_{i}\right. \\
& \left.+\left(\gamma_{e}-1\right)\left(i(\mathbf{k} \cdot \mathbf{b})^{2} c_{e}^{2}+\alpha v_{e n} \mathbf{V}_{e 0} \cdot \mathbf{b b} \cdot \mathbf{k}\right) / \Omega_{e}\right\}=0
\end{aligned}
$$

where $\gamma_{e}=1+t_{1} / n_{1}$ is the ratio of the principal specific heats for the electron gas and is given by 


$$
\begin{aligned}
\gamma_{e}-1= & \left\{i \omega_{e}+\left(2 v_{e n} \Omega_{e} /\left(\left(v_{e n}-i \omega_{e}\right) k^{2} c_{e}^{2} \Omega_{i}\right)\right)\right. \\
& \times\left(\mathbf{V}_{e 0} \cdot \mathbf{b b} \cdot \mathbf{k}\left(\omega\left(v_{i n}-i \omega\right)+i k^{2} c^{2}\right)\right\} \\
& \div\left\{1.5 i \omega_{e}-2.5(\mathbf{b} \cdot \mathbf{k})^{2} c_{e}^{2} /\left(v_{e n}-i \omega_{e}\right)-\delta_{e}^{*} v_{e n}\right. \\
& -\left(2 v_{e n} /\left(v_{e n}-i \omega_{e}\right) c_{e}^{2}\right)\left(i \mathbf{V}_{e 0} \cdot \mathbf{b b} \cdot \mathbf{k} c_{e}^{2}+\alpha v_{e n}\left(\mathbf{V}_{e 0} \cdot \mathbf{b}\right)^{2}\right\}
\end{aligned}
$$

Also in Eqs. (15) and (16), $c=\sqrt{\left(T_{e 0}+T_{i 0}\right) / m_{i}}$ and $c_{e}=\sqrt{T_{e 0} / m_{e}}$ are respectively the ion acoustic and electron thermal speeds.

Before proceeding to the low frequency thermal instability, in the next section, it is worth demonstrating at this point that there is no instability if the waves, described by Eqs. (15) and (16), are isothermal. Isothermal fluctuations imply $\gamma_{e}=1$. It may also be assumed in what follows, without any significant loss of generality, that the steady electron drift is exactly parallel to the geomagnetic field. Then, for isothermal waves, Eq. (15) becomes

$$
\begin{aligned}
\omega & +\omega_{e} v_{e n} \Omega_{i} /\left(v_{i n} \Omega_{e} \cos ^{2} \theta\right) \\
& -\left(i / v_{i n}\right)\left(\omega^{2}+\omega_{e}^{2} \Omega_{i} /\left(\Omega_{e} \cos ^{2} \theta\right)-k^{2} c^{2}\right)
\end{aligned}
$$

This dispersion relation is also derivable as the fluid limit of the full kinetic theory detailed by Lee et al. (1971) (also see the Appendix).

Explicitly introducing a complex wave frequency i.e. $\omega \rightarrow \omega+i \gamma$, where $\gamma$ is the growth rate, and separating the dispersion relation Eq. (17) into real and imaginary parts then yields two conditions, which are, to a good approximation

$\omega=k V_{e 0} v_{e n} \Omega_{i} /\left(v_{i n} \Omega_{e} \cos \theta\right)$

and

$\gamma=k^{2}\left(V_{e 0}^{2} \Omega_{i} / \Omega_{e}-c^{2}\right) / v_{i n}$

In Eqs. (18) and (19) the relation $\omega_{e}=\omega-k V_{e 0} \cos \theta$ and the fact that, in the lower ionosphere, $\Omega_{i} v_{e n} \ll \Omega_{e} v_{i n}$ and hence $\left|\omega_{e}\right| \gg|\omega|$, have been utilised. Notice these inequalities also imply that, unlike the FBI case where ion inertia dominates electron inertia in the destabilisation process, here it is the electron inertia which dominates.

It must now be pointed out that, even though it has been assumed above that the waves are isothermal, steady heating of the electron gas cannot be neglected and hence, from Eqs. (3) and (4), under steady conditions and in the absence of gradients

$T_{e 0}=T_{i 0}+m_{e} V_{e 0}^{2} / \delta_{e}$

In Eq. (20), it is assumed that the ions are unheated and that in the limit of zero electron flow the electrons and ions are in thermal equilibrium. Recalling the definition of the ion acoustic speed, Eq. (19) should thus be replaced by

$\gamma=k^{2}\left(V_{e 0}^{2}\left(1-\delta_{e}^{-1}\right) \Omega_{i} / \Omega_{e}-c_{0}^{2}\right) / v_{\text {in }}$

where $c_{0}$ is the ion acoustic speed with equal electron and ion temperatures. Further, noting that $\delta_{e} \ll 1$ (Gurevich, 1978), implies that $\gamma$ is entirely negative and the waves are always damped. Hence, there is no instability under isothermal conditions. The reason for this is clear from Eqs. (19) and (21). In order for the instability to arise the destabilising effects of electron inertia, the first term on the right hand side of Eqs. (19) and (21), has to exceed the effect of thermal pressure gradients, the remaining terms on the right hand side of Eqs. (19) and (21). These cause damping when the pressure perturbations are in phase with the density perturbations, which they necessarily are in the isothermal case where the pressure perturbation is just $n_{1} T_{e 0}$. Thus, as the electron velocity increases, the destabilising effects always lag behind the stabilising ones, in the isothermal case. The situation is entirely different in the non isothermal low frequency case, dealt with later.

\section{A low frequency thermal instability}

The dispersion relation above simplifies considerably under low frequency conditions, when

$\left|\delta_{e} v_{e n}\right| \gg\left|\omega_{e}\right|$ and $\left|2.5 k^{2} c_{e}^{2} \cos ^{2} \theta /\left(v_{e n}-i \omega_{e}\right)\right|$.

Physically, these conditions mean that electron inertia and electron thermal conductivity are neglected compared to inelastic collisional heat loss. Equation (22) also implies $\left|\omega_{e}\right| \ll\left|v_{e n}\right|$. Under these conditions, then the relations (15) and (16) simplify to

$$
\begin{aligned}
& \omega+\left(\omega_{e}+\alpha\left(\gamma_{e}-1\right) k V_{e 0} \cos \theta\right)\left(v_{e n} \Omega_{i} /\left(v_{i n} \Omega_{e} \cos ^{2} \theta\right)\right) \\
& -\left(i / v_{i n}\right)\left(\omega^{2}+\omega_{e}^{2} \Omega_{i} /\left(\Omega_{e} \cos ^{2} \theta\right)\right. \\
& \left.-k^{2}\left(c^{2}+\left(\gamma_{e}-1\right) c_{e}^{2} \Omega_{i} / \Omega_{e}\right)\right)=0
\end{aligned}
$$

and

$\gamma_{e}-1=-2 \Omega_{e} V_{e 0} \omega v_{i n} /\left(k c_{e}^{2} \Omega_{i} v_{e n}\left(\delta_{e}^{*}+2 \alpha V_{e 0}^{2} / c_{e}^{2}\right)\right)$.

Solving Eqs. (23) and (24) simultaneously for $\omega+i \gamma$ and $\gamma_{e}$ leads, to a good approximation, to

$$
\begin{aligned}
\gamma_{e}= & 1-2 V_{e 0}^{2} /\left(c_{e}^{2} \delta_{e}^{*}\right) \\
= & 1-2\left(\delta_{e} / \delta_{e}^{*}\right)\left(T_{e 0}-T_{i 0}\right) / T_{e 0} \\
\omega= & \left(k V_{e 0} v_{e n} \Omega_{i} / v_{i n} \Omega_{e} \cos \theta\right) \\
& \times\left(1+2 \alpha\left(\delta_{e} / \delta_{e}^{*}\right)\left(T_{e 0}-T_{i 0}\right) / T_{e 0}\right) \\
\gamma= & \left(k^{2} / v_{i n} m_{i}\right)\left(2\left(T_{e 0}-T_{i 0}\right)\left(\delta_{e} / \delta_{e}^{*}\right)-\left(T_{e 0}+T_{i 0}\right)\right) \\
= & -\left(k^{2} / v_{i n} m_{i}\right)\left(\gamma_{e} T_{e 0}+T_{i 0}\right)
\end{aligned}
$$

In Eqs. (25) to (27), relation (20) has been utilised. The condition for instability $(\gamma>0)$ is easily obtained from Eq. (27), with the aid of Eq. (13) and the approximation in the range $T_{i 0}<T_{e 0}<4 T_{i 0}$ (Gurevich, 1978)

$\delta_{e}^{*} / \delta_{e}=1-\left(T_{e 0}-T_{i 0}\right) /\left(3 T_{i 0}\right)$.

Then, the condition for instability requires

$T_{e 0}=T_{i 0}+m_{e} V_{e 0}^{2} / \delta_{e}>2 T_{i 0}$.

Thus, when the electron temperature is elevated to over twice the ion temperature by the heating effect of the 
field aligned electron drift, the thermal instability of low frequency waves arises. It should be noticed that the threshold condition is independent of the propagation angle, $\theta$. Thus, within the limits set by condition (14), the values of the growth rates are isotropic, even though the phase speeds, given by Eq. (26), increase with $(\cos \theta)^{-1}$. It is also useful to write the threshold criterion for the instability in terms of the electron flow speed. From Eq. (29), this is just

$V_{e 0}>\sqrt{\delta_{e} T_{i 0} / m_{e}}$.

The phase velocity for marginally unstable waves, i.e. with the inequality in Eq. (31) replaced by equality, is then

$\omega / k=c \sqrt{\delta_{e} \Omega_{i} / 3 \Omega_{e}}\left(v_{e n} / v_{i n}\right)(1+3 \alpha / 2) / \cos \theta$.

Although the two collision frequencies in Eq. (31) are each strong functions of altitude, through their dependence on the neutral density, their ratio is almost constant with altitude. The other background parameters are only weakly dependent on height, if at all. Consequently, the threshold values of the flow speed of the electrons and the wave phase speeds are almost independent of altitude, in the E-region. Typical values of the relevant parameters for an altitude of $100 \mathrm{~km}$ are approximately (Gurevich, 1978)

$$
\begin{aligned}
& v_{e n} \approx 1 \times 10^{5} \mathrm{~s}^{-1}, \quad v_{\text {in }} \approx 5 \times 10^{3} \mathrm{~s}^{-1}, \\
& \Omega_{e} \approx 9 \times 10^{6} \mathrm{rad} \mathrm{s}^{-1}, \quad \Omega_{i} \approx 160 \mathrm{rad} \mathrm{s}^{-1}, \\
& \delta_{e} \approx 5 \times 10^{-3}, \quad \alpha=5 / 6, \quad T_{i 0} \approx 200 \mathrm{~K} .
\end{aligned}
$$

Inserting these into Eqs. (30) and (31) yields a threshold speed and a wave phase speed of $3.5 \mathrm{~km} \mathrm{~s}^{-1}$ and $1.5 \mathrm{~m} \mathrm{~s}^{-1}$ respectively. Thus, the threshold value of the field aligned electron drift speed is well below the values previously proposed by Chaturvedi et al. (1987) and Villain et al. (1987). The wave phase speed is remarkably low compared with a typical ion acoustic speed of $\sim 250 \mathrm{~m} \mathrm{~s}^{-1}$ and implies, since velocities have been taken with respect to the background neutrals, that the observed wave phase speed will be essentially equal to the neutral wind component along the propagation direction.

Finally, in this section, the physical basis of the low frequency instability can be fairly straightforwardly understood in terms of the relationship between the temperature and density perturbations. This is defined by the ratio of the principal specific heats, of the electron gas, $\gamma_{e}=1+t_{1} / n_{1}$, given by Eq. (25). The condition for instability (29) is equivalent to requiring $\gamma_{e}$ to be negative and smaller than the ratio of the ion to electron temperature (see Eq. 27). This happens when the relative temperature perturbations are of larger amplitude and in antiphase with the relative plasma density perturbations. The antiphase between the temperature and density perturbations occurs because the temperature is in phase with the electron velocity perturbations in the low frequency approximation, through the heating term, whilst the density is in antiphase with the electron velocity through the continuity equation (note that $\omega_{e}$ is always negative). The consequence of these effects, in contrast to the isothermal case treated in Sect. 2, is to make the density perturbations in antiphase with the total pressure perturbations. In this case the pressure gradients act to destabilise rather than stabilise the plasma.

This description of the physical basis of the instability is similar to that for the DSI (Dimant and Sudan, 1995, 1997; Robinson, 1998). The chief difference between the DSI and the present theory lies in the geometry of the electron velocity in the two cases. As a consequence it is the modulation of heating due to the electron Pedersen current which is responsible for the DSI, whereas the modulation of heating due to the electron field aligned current is responsible for the new instability described above. However, the characteristics of the destabilised waves in the two cases are clearly very different.

\section{Observational implications}

The analysis of the previous section is briefly examined in a quantitative manner, in order to establish its observational implications. Having determined the approximate values of the threshold electron field aligned drift and the phase speed of the unstable waves in the previous section it is important to establish the range $\mathbf{k}$ vector characteristics for which the instability occurs. The results will be altitude dependent through the altitude dependence of the electron-neutral collision frequency and conditions (14) and (22). Substituting the expression for the threshold velocity from Eq. (30) into Eq. (22) yields, for the limiting wavelength

$\lambda \gg 2 \pi \sqrt{\delta_{e} T_{i 0} / m_{e}} / v_{e n}$.

The altitude range of interest is $90-100 \mathrm{~km}$. At $100 \mathrm{~km}$ altitude, using the typical parameter values from Sect. 3, leads approximately to a propagation angle with respect to the geomagnetic field of less than $89^{\circ}$ and a wavelength much greater than $50 \mathrm{~m}$. At $90 \mathrm{~km}$, where the collision frequencies are about an order of magnitude higher, the corresponding limits are $84^{\circ}$ and $5 \mathrm{~m}$ respectively. Clearly, within the height range of interest the instability operates over a wide range of angles to the geomagnetic field, but the instability is restricted to rather large wavelengths. At the higher end of the altitude range, in particular, the wavelengths associated with the instability are too long for radar backscatter with conventional VHF radars. Whilst HF radars operating at frequencies below $10 \mathrm{MHz}$, which scatter from waves with wavelengths above $15 \mathrm{~m}$, could probably detect some of these unstable waves in this height range, Rüster and Schlegel (1999) made their observations with the ALOMAR SOUSY radar operating at $50 \mathrm{MHz}$ which scatters from $3 \mathrm{~m}$ irregularities. This is outside the range for unstable waves at all the heights considered, although it comes fairly close at $90 \mathrm{~km}$. However, the angular range of Rüster and Schlegel's (1999) observations is well within the unstable limits. Their results are also consistent with the very low phase 
velocities which are characteristic of the instability discussed already.

\section{Conclusions}

The theory outlined, in which moderate field aligned electron drifts can destabilise very large aspect angle plasma irregularities in the lower ionosphere through electron-neutral collisional heating effects, has the following main characteristics, in the fluid limit:

1. The threshold electron drifts parallel to the geomagnetic field are a few $\mathrm{km} \mathrm{s}^{-1}$ and are independent of the angle, $\theta$, between the wave propagation direction and the magnetic field, for $\theta<80^{\circ}$, approximately.

2. The phase speed of the marginally unstable waves is a few $\mathrm{m} \mathrm{s}^{-1}$, along the geomagnetic field and increases as $\sec \theta$.

3. The values of the threshold flow speeds and phase speeds above is largely independent of altitude between $90-100 \mathrm{~km}$.

4. The instability occurs at wavelengths longer than a characteristic scale which is altitude dependent with a value of around $5 \mathrm{~m}$ at $90 \mathrm{~km}$ and $50 \mathrm{~m}$ at $100 \mathrm{~km}$.

These results constitute an important new instability in the lower E-region. Although some of its characteristics (particularly points 1 to 3 ) are strikingly similar to the recent observations reported by Rüster and Schlegel (1999), the present theory, as it stands, cannot directly account for the short wavelengths involved, particularly at $100 \mathrm{~km}$. Consequently an entirely different mechanism, from the one outlined here, cannot be ruled out at this stage. However, it is worth considering, albeit briefly, how the wavelength range of the present theory might be extended to lower values.

Two fairly straightforward extensions are readily apparent. Firstly, it is possible that the electron momentum collisional loss rate could be enhanced above that provided by electron-neutral collisions alone due to some anomalous process. Anomalous collision processes have been commonly invoked elsewhere (e.g. Sudan, 1983; Robinson, 1986, 1998; Hamza, 1992; Mishin et al., 1992). The mechanisms involved vary, but the essential feature is that very short scale waves randomly scatter the electrons and effectively enhance the electron momentum loss rate. Hamza (1992) has suggested values of anomalous collision frequency up to two orders of magnitude above those due to electronneutral collisions alone. If the electron collision frequency were raised in this way to values of a few times $10^{6} \mathrm{~s}^{-1}$, then the wavelength range of the instability could be brought within the range of the observations of Rüster and Schlegel (1999). It should be noted that magnetised electrons are not essential for the instability to work so there is no difficulty in the electron collision frequency approaching the electron gyro frequency. In this case, however, $\Omega_{e}$ would replaced by $\sqrt{\Omega_{e}^{2}+v_{e n}^{2}}$ in equations of motion of the electron. However, a more serious limitation of increasing collisions is the destruction of the directed electron motion through the randomising effect of the collisions. Detailed consideration of this important topic is beyond the scope of the present study but it has been discussed extensively by Rietveld et al. (1991). These authors argue that field parallel electron currents can penetrate into the lower, collisional, ionosphere due to current continuity effects and polarisation electric fields arising parallel to the geomagnetic field. Such processes would be more likely to occur in the absence of alternative current paths such as those proved across the geomagnetic fields by $\mathbf{E} \times \mathbf{B}$ drifts in the upper E-region. These arise in the presence of convection electric fields of magnetospheric origin and would have the effect of shorting out the electric field along the magnetic field lines.

In invoking anomalous collisions in the present context, the obvious question of their origin arises. One possibility is field parallel effects produced by obliquely propagating short scale FBI waves, at least at the higher altitude range, above about $95 \mathrm{~km}$ (Hamza and St.-Maurice, 1995). These waves would also heat the plasma, which might allow the very short wavelength $(<10 \mathrm{~cm})$, effectively collisionless, ion acoustic waves to be destabilised by the field parallel, kinetic ion acoustic instability (Kindel and Kennel, 1971). These short scale ion acoustic waves would then give rise to enhanced anomalous collisions for the longer wavelength fluid waves under consideration here.

A second mechanism which would extend the wavelength range of the long wavelength thermal instability to lower values involves the nonlinear development of the electron density, temperature and velocity perturbations. If an initially quasi-sinusoidal disturbance, with a characteristic scale of several tens of metres along the geomagnetic field, began to grow, eventually the electron temperature crests, which coincides with the velocity crests and the density troughs, would increase the local electron temperature and begin to lower the threshold for shorter wavelengths. This would also have the effect of sharpening the temperature crests and the density troughs. The temperature troughs would lead to a locally lower electron temperatures which would tend to stabilise the instability and lead to flatter temperature troughs. The sharpening temperature peaks and resultant sharper density troughs would cause backscatter at shorter wavelengths than the original quasi-sinusoidal disturbance. Note also that the mean temperature measured along the geomagnetic field direction by, say, incoherent scatter radar, would not deviate much from the undisturbed background temperature.

To conclude, then, it appears possible that moderate field aligned electron flows can destabilise waves in the lower E-region of the high-latitude ionosphere, with very little aspect angle dependence, as long as their wavelengths are sufficiently long. Typically these wavelengths need to be at least a few tens of metres in the altitude range $90-100 \mathrm{~km}$. Such waves could enhance the backscatter cross sections of the lower E-region for $\mathrm{HF}$ radars operating at frequencies below $10 \mathrm{MHz}$. Nonlinear effects may reduce the limiting wavelength of the instability, so that radars operating at higher frequencies may also become sensitive to the instability. 
Given the more favourable sensitivity of HF over VHF radars to this new instability, it may seem surprising that no HF radar observation of non aspect sensitive scatter has so far been reported in the literature. However two points should be remembered in this context. First, all of the major HF coherent radar systems operating at high latitudes, such as SuperDARN (Greenwald et al., 1995) are designed to detect scatter from field aligned irregularities and thus have beams directed preferentially in directions orthogonal to the geomagnetic field. Other HF systems, such as ionosondes, which do have broad field aligned beams, are probably not sensitive enough to detect the kind of scatter reported by Rüster and Schlegel (1999). Second, it may well be that auroral non aspect sensitive scatter has been detected but not recognised. Such scatter could occur, for example, in the near ranges of SuperDARN radars, whose beams are in fact broad enough to detect backscatter at large aspect angles in the D- and E-regions. However, such scatter would easily be confused with meteor scatter from these altitudes. Meteor scatter is a common feature of near range backscatter from the SuperDARN radars (Hall et al., 1997) and comes from targets which are clearly not in general field aligned. Furthermore, these targets move with the background wind, just as predicted for the new instability. On the basis of the theory presented, therefore, it would probably be worthwhile re-examining these near range scatter data in the lower ionosphere for evidence of any association with field aligned currents.

Acknowledgements. The Editor-in-chief thanks J. P. Villain for his help in evaluating this paper.

\section{Appendix: kinetic theory and the limits of the collisionless and fluid approximations}

The general linear kinetic theory dispersion relation which is applicable in a collisional partially ionised plasma to the case of magnetised electrons and unmagnetised ions has the form (Lee et al., 1971)

$$
\begin{aligned}
k^{2} \lambda_{D}^{2} & +\frac{T_{e}}{T_{i}} \frac{1+\frac{\omega+i v_{i n}}{\sqrt{2} k c_{i}} Z_{i}}{1+\frac{i v_{i n}}{\sqrt{2} k c_{i}} Z_{i}} \\
+ & \frac{1+\frac{\omega_{e}+i v_{e n}}{\sqrt{2} k c_{e}} \exp \left(-\mu_{e}\right) \sum_{r} I_{r}\left(\mu_{e}\right) Z_{e r}}{1+\frac{i v_{e n}}{\sqrt{2} k c_{e}} \exp \left(-\mu_{e}\right) \sum_{r} I_{r}\left(\mu_{e}\right) Z_{e r}}=0
\end{aligned}
$$

where, $c_{i}=\sqrt{T_{i 0} / m_{i}}$ and

$$
\begin{aligned}
& Z_{i}=Z\left(\frac{\omega+i v_{i n}}{\sqrt{2} k c_{i}}\right) \\
& Z_{e r}=Z\left(\frac{\omega_{e}+i v_{e n}-r \Omega_{e}}{\sqrt{2} k c_{e} \cos \theta}\right)
\end{aligned}
$$

and

$$
\mu_{e}=\left(k c_{e} \sin \theta / \sqrt{2} \Omega_{e}\right)^{2} .
$$

Also, here, $\lambda_{D}$ is the Debye length, $Z$ is the plasma dispersion function (Fried and Conte, 1961) and $I_{r}\left(\mu_{e}\right)$ is a modified Bessel function of order $r$. It is sufficient to take only cases where the wavelength is long compared to the Debye length and the electron wave frequency is small compared to the electron gyrofrequency. Then Eq. (A.1) reduces to

$$
\frac{T_{e}}{T_{i}} \frac{1+\frac{\omega+i v_{i n}}{\sqrt{2} k c_{i}} Z_{i}}{1+\frac{i v_{i n}}{\sqrt{2} k c_{i}} Z_{i}}+\frac{1+\frac{\omega_{e}+i v_{e n}}{\sqrt{2} k c_{e}} Z_{e 0}}{1+\frac{i v_{e n}}{\sqrt{2} k c_{e}} Z_{e 0}}=0 .
$$

The fluid limit of Eq. (A.5) is obtained for the case where the size of the arguments in $Z_{e 0}$ and $Z_{i}$ are large compared to 1 . Then $Z$ is approximated by an asymptotic expansion (Fried and Conte, 1961) as

$Z(x)=-\left(1+1 / 2 x^{2}\right) / x$

Under this approximation, together with the condition Eq. (14), Eq. (A.5) becomes identical with the isothermal fluid wave Eq. (17).

The fluid limit thus corresponds essentially to the condition $v_{i n} \gg k c_{i}$ and $v_{e n} \gg k c_{e}$. The electron condition provides the constraint in this case and requires the wavelengths of interest to greatly exceed $3.5 \mathrm{~m}$ at $100 \mathrm{~km}$ and around $0.35 \mathrm{~m}$ at $90 \mathrm{~km}$. These conditions are well satisfied by the long wavelength thermal instability derived in Sect. 3.

For effectively collisionless conditions to be applicable, these inequalities are reversed. Then the ion constraint is the more important and requires the wavelengths to be much less than $0.3 \mathrm{~m}$ at $100 \mathrm{~km}$ and much less than $0.03 \mathrm{~m}$ at $90 \mathrm{~km}$. Thus, these wavelength constraints apply to the excitation of the collisionless kinetic ion acoustic instability in the altitude range 90 $100 \mathrm{~km}$.

\section{References}

Buneman, O., Excitation of field aligned sound waves by electron streams, Phys. Rev. Lett., 10, 285-287, 1963.

Bythrow, P. F., T. A. Potemra, W. B. Hanson, L. J. Zanetti, C.-I. Meng, R. E. Huffman, F. J. Rich, and D. A. Hardy, Earthward directed high-density Birkland currents observed by HILAT, J. Geophys. Res., 89, 9114-9118, 1984.

Chaturvedi, P. I., J. D. Huba, S. L. Ossakow, P. Satyanarayana, and J. A. Fedder, Parallel current effects on two-stream electrojet plasma instabilities, J. Geophys. Res., 92, 8700-8706, 1987.

Dimant, Y. S., and R. N. Sudan, Kinetic theory of the FarleyBuneman instability in the E-region of the ionosphere, J. Geophys. Res., 100, 14 605-14 623, 1995.

Dimant, Y.S., and R. N. Sudan, Physical nature of a new cross-field current-driven instability in the lower ionosphere, J. Geophys. Res., 102, 2551-2563, 1997.

Farley D. T., A Plasma instability resulting in field-aligned irregularities in the ionosphere, J. Geophys. Res., 68, 60836097, 1963.

Fried, B. D., and S. D. Conte, The plasma dispersion function, Academic Press, New York, 1961.

Golant, V. E., A. P. Zhilinsky, and I. E. Sakharov, Fundamentals of plasma physics, Wiley, New York, 1980.

Greenwald, R. A., K. B. Baker, J. R. Dudeney, M. Pinnock, T. B. Jones, E. C. Thomas, J.-P. Villain, J.-C. Cerisier, C. Senior, 
C. Hanuise, R. D. Hunsucker, G. Sofko, J. Koehler, E. Nielsen, R. Pellinen, A. D. M. Walker, N. Sato, and H. Yamagashi, DARN/SuperDARN: a global view of the dynamics of highlatitude convection, Space Sci. Rev., 71, 761-796, 1995.

Gurevich, A. V., Nonlinear phenomena in the ionosphere, Springer, New York, Berlin Heidelberg 1978.

Haldoupis, C., J. A. Koehler, G. J. Sofko, J. Mu, D. André, A. V. Kustov, and D. W. Danskin, Auroral radar frequency broadening measurements at large aspect angles and comparison with theory, J. Geophys. Res., 99, 8925-8935, 1994.

Hall, G. E., J. W. MacDougall, D. R. Moorecroft, and J.-P. St.Maurice, Super Dual Auroral Radar Network observations of meteor scatter, J. Geophys. Res., 102, 14 603-14 614, 1997.

Hamza, A. M., A nonlinear theory for large aspect angle echoes in the auroral E region, J. Geophys. Res., 97, 16981-16993, 1992.

Hamza, A. M., and J.-P. St.-Maurice, A fully self-consistent fluid theory of anomalous transport in Farley-Buneman turbulence, J. Geophys. Res., 100, 9653-9668, 1995.

Kindel, J. M., and C. F. Kennel, Topside current instabilities, J. Geophys. Res., 76, 3055-3078, 1971.

Lee, K., C. F. Kennel, and J. M. Kindel, High frequency Hall current instability, Radio Sci., 6, 209-213, 1971.
Mishin, E. V., L. N. Lukjanova, S. F. Marenko, and B. M. Atamanjuk, Radio wave dissipation in turbulent auroral plasma during the precipitation of energetic electrons, Radio Sci., 27, 283-287, 1992.

Rietveld, M. T., P. N. Collis, and J.-P. St.-Maurice, Naturally enhanced ion acoustic waves in the auroral ionosphere observed with the EISCAT $933 \mathrm{MHz}$ radar, J. Geophys. Res., 96, 19291$19305,1991$.

Robinson, T. R., Towards a self consistent theory of radar backscatter, J. Atmos. Terr. Phys., 48, 417-422, 1986.

Robinson, T. R., The effects of small scale field aligned irregularities on E-region conductivities: implications for electron thermal processes, Adv. Space Sci., 22, 1357-1360, 1998.

Rüster, R., and K. Schlegel, Non-magnetic aspect sensitive auroral echoes from the lower E region observed at $50 \mathrm{MHz}$, Ann.Geophysicae, 17, 1284-1292, 1999.

Sudan, R. N., Unified theory of type-I and type-II irregularities in the equatorial electrojet, J. Geophys. Res., 88, 4853-4860, 1983.

Villain, J.-P., R. A. Greenwald, K. B. Baker, and J. M. Ruohonemi, $\mathrm{HF}$ radar observations of $\mathrm{E}$ region plasma irregularities produced by oblique electron streaming, J. Geophys. Res., 92, $12327-12342,1987$. 